
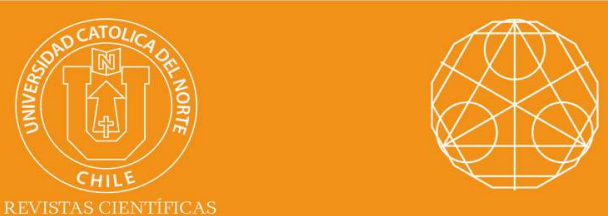

\title{
Some hyperstability results for a Cauchy-Jensen type functional equation in 2-Banach spaces
}

\section{Khaled Yahya Naif Sayar ${ }^{1}$}

Amal Bergam ${ }^{2}$

University Abdelmalek Essaadi, Polydisciplinary Faculty of Larache, MAE2D laboratort, Larache, Morocco

1ఐ khaledsayar@gmail.com ; 2 bergamamal11@gmail.com

Received: December 2018 | Accepted: March 2019

\section{Abstract:}

In this paper, we investigate some stability and hyperstability results for the following Cauchy-Jensen functional equation

$$
f\left(\frac{x+y}{2}+z\right)+f\left(\frac{x-y}{2}+z\right)=f(x)+2 f(z)
$$

in 2-Banach spaces by using Brzdȩk's fixed point approach.

Keywords: Stability; Hyperstability; 2-Banach space; CauchyJensen functional equation.

MSC (2010): 39B82; 39B52; 47H10.

\section{Cite this article as (IEEE citation style):}

K. Y. Naif Sayar and A. Bergam, "Some hyperstability results for a Cauchy-Jensen type functional equation in 2 Banach spaces", Proyecciones (Antofagasta, On line), vol. 39, no. 1, pp. 73-89, Feb. 2020, doi: 10.22199/issn.07176279-2020-01-0005. [Accessed dd-mm-yyyy].

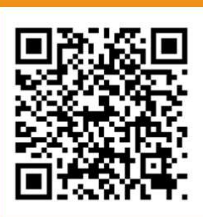

Article copyright: (C) 2020 Khaled Yahya Naif Sayar and Amal Bergam.This is an open access article distributed under the terms of the Creative Commons Licence, which permits unrestricted use and distribution provided the original author and source are credited. 


\section{Introduction}

Let $E, Y$ be normed spaces. A function $f: E \rightarrow Y$ is Cauchy-Jensen provided it satisfies the functional equation

$$
f\left(\frac{x+y}{2}+z\right)+f\left(\frac{x-y}{2}+z\right)=f(x)+2 f(z) \text { for all } x, y, z \in E,
$$

and we can say that $f: E \rightarrow Y$ is Cauchy-Jensen on $E_{0}$ if it satisfies (1.1) for all $x, y, z \in E_{0}$ such that $\frac{x+y}{2}+z \neq 0$ and $\frac{x-y}{2}+z \neq 0$.

Recently, interesting results concerning the Cauchy-Jensen functional equation (1.1) have been obtained in [7] and [20].

Throughout this paper, we will denote the set of natural numbers by $\mathbf{N}, \mathbf{N}_{0}:=\mathbf{N} \cup\{0\}$ and the set of real numbers by $\mathbf{R}$. By $\mathbf{N}_{m}, m \in \mathbf{N}$, we will denote the set of all natural numbers greater than or equal to $m$.

Let $\mathbf{R}_{+}=[0, \infty)$ the set of nonnegative real numbers. We write $B^{A}$ to mean the family of all functions mapping from a nonempty set $A$ into a nonempty set $B$ and we use the notation $E_{0}$ for the set $E \backslash\{0\}$.

We need to recall some basic facts concerning 2-normed spaces and some preliminary results (see, for instance, [17]).

Definition 1.1. let $X$ be a real linear space with $\operatorname{dim} X>1$ and $\|\cdot, \cdot\|$ : $X \times X \longrightarrow \mathbf{R}_{+}$be a function satisfying the following properties:

1. $\|x, y\|=0$ if and only if $x$ and $y$ are linearly dependent,

2. $\|x, y\|=\|y, x\|$,

3. $\|\lambda x, y\|=|\lambda|\|x, y\|$,

4. $\|x, y+z\| \leq\|x, y\|+\|x, z\|$,

for all $x, y, z \in X$ and $\lambda \in \mathbf{R}$. Then the function $\|\cdot, \cdot\|$ is called a 2-norm on $X$ and the pair $(X,\|\cdot, \cdot\|)$ is called a linear 2-normed space. Sometimes the condition (4) called the triangle inequality. 
Example 1.2. For $x=\left(x_{1}, x_{2}\right), y=\left(y_{1}, y_{2}\right) \in E=\mathbf{R}^{2}$, the Euclidean 2-norm $\|x, y\|_{E}$ is defined by

$$
\|x, y\|_{E}=\left|x_{1} y_{2}-x_{2} y_{1}\right|
$$

Definition 1.3. A sequence $\left\{x_{k}\right\}$ in a 2-normed space $X$ is called a convergent sequence if there is an $x \in X$ such that

$$
\lim _{k \rightarrow \infty}\left\|x_{k}-x, y\right\|=0
$$

for all $y \in X$. If $\left\{x_{k}\right\}$ converges to $x$, write $x_{k} \longrightarrow x$ with $k \longrightarrow \infty$ and call $x$ the limit of $\left\{x_{k}\right\}$. In this case, we also write $\lim _{k \rightarrow \infty} x_{k}=x$.

Definition 1.4. A sequence $\left\{x_{k}\right\}$ in a 2-normed space $X$ is said to be a Cauchy sequence with respect to the 2-norm if

$$
\lim _{k, l \rightarrow \infty}\left\|x_{k}-x_{l}, y\right\|=0
$$

for all $y \in X$. If every Cauchy sequence in $X$ converges to some $x \in X$, then $X$ is said to be complete with respect to the 2-norm. Any complete 2-normed space is said to be a 2-Banach space.

Next, it is easily seen that we have the following property.

Lemma 1.5. If $X$ is a linear 2-normed space, $x, y_{1}, y_{2} \in X, y_{1}, y_{2}$ are linearly independent, and

$$
\left\|x, y_{1}\right\|=\left\|x, y_{2}\right\|=0
$$

then $x=0$.

Let us yet recall a lemma from [19].

Lemma 1.6. If $X$ is a linear 2-normed space and $\left(x_{n}\right)_{\in \mathbf{N}}$ is a convergent sequence of elements of $X$, then

$$
\lim _{n \rightarrow \infty}\left\|x_{n}, y\right\|=\left\|\lim _{n \rightarrow \infty} x_{n}, y\right\|=0, \quad y \in X
$$


The problem of the stability of functional equations was first raised by Ulam [21]. This included the following question concerning the stability of group homomorphisms.

Let $\left(G_{1}, *_{1}\right)$ be a group and let $\left(G_{2}, *_{2}\right)$ be a metric group with a metric $d(.,$.$) . Given \varepsilon>0$, does there exists $a \delta>0$ such that if a mapping $h: G_{1} \rightarrow G_{2}$ satisfies the inequality

$$
d\left(h\left(x *_{1} y\right), h(x) *_{2} h(y)\right)<\delta
$$

for all $x, y \in G_{1}$, then there exists a homomorphism $H: G_{1} \rightarrow G_{2}$ with

$$
d(h(x), H(x))<\varepsilon
$$

for all $x \in G_{1}$ ?

If the answer is affirmative, we say that the equation of homomorphism

$$
h\left(x *_{1} y\right)=h(x) *_{2} H(y)
$$

is stable.

The first partial answer to Ulam's question was given by Hyers [18] and he established the stability result as follows:

Theorem 1.7. [18] Let $E_{1}$ and $E_{2}$ be two Banach spaces and $f: E_{1} \rightarrow E_{2}$ be a function such that

$$
\|f(x+y)-f(x)-f(y)\| \leq \delta
$$

for some $\delta>0$ and for all $x, y \in E_{1}$. Then the limit

$$
A(x):=\lim _{n \rightarrow \infty} 2^{-n} f\left(2^{n} x\right)
$$

exists for each $x \in E_{1}$, and $A: E_{1} \rightarrow E_{2}$ is the unique additive function such that

$$
\|f(x)-A(x)\| \leq \delta
$$

for all $x \in E_{1}$. Moreover, if $f(t x)$ is continuous in $t$ for each fixed $x \in E_{1}$, then the function $A$ is linear. 
Later, T. Aoki [4] and D. G. Bourgin [8] considered the problem of stability with unbounded Cauchy differences. Th. Rassias [21] attempted to weaken the condition for the bound of the norm of Cauchy difference

$$
\|f(x+y)-f(x)-f(y)\|
$$

and proved a generalization of Theorem 1.7 using a direct method (cf. Theorem 1.8):

Theorem 1.8. [24] Let $E_{1}$ and $E_{2}$ be two Banach spaces. If $f: E_{1} \rightarrow E_{2}$ satisfies the inequality

$$
\|f(x+y)-f(x)-f(y)\| \leq \theta\left(\|x\|^{p}+\|y\|^{p}\right)
$$

for some $\theta \geq 0$, for some $p \in \mathbf{R}$ with $0 \leq p<1$, and for all $x, y \in E_{1}$, then there exists a unique additive function $A: E_{1} \rightarrow E_{2}$ such that

$$
\|f(x)-A(x)\| \leq \frac{2 \theta}{2-2^{p}}\|x\|^{p}
$$

for each $x \in E_{1}$. If, in addition, $f(t x)$ is continuous in $t$ for each fixed $x \in E_{1}$, then the function $A$ is linear.

Later, Th. Rassias [22],[23] motivated Theorem 1.8 as follows:

Theorem 1.9. [22],[23] Let $E_{1}$ be a normed space, $E_{2}$ be a Banach space, and $f: E_{1} \rightarrow E_{2}$ be a function. If $f$ satisfies the inequality

$$
\|f(x+y)-f(x)-f(y)\| \leq \theta\left(\|x\|^{p}+\|y\|^{p}\right)
$$

for some $\theta \geq 0$, for some $p \in \mathbf{R}$ with $p \neq 1$, and for all $x, y \in E_{1}-\left\{0_{E_{1}}\right\}$, then there exists a unique additive function $A: E_{1} \rightarrow E_{2}$ such that

$$
\|f(x)-A(x)\| \leq \frac{2 \theta}{\left|2-2^{p}\right|}\|x\|^{p}
$$

for each $x \in E_{1}-\left\{0_{E_{1}}\right\}$.

Note that Theorem 1.9 reduces to Theorem 1.7 when $p=0$. For $p=1$, the analogous result is not valid. Also, J. Brzdęk [9] showed that estimation (1.3) is optimal for $p \geq 0$ in the general case.

Recently, J. Brzdęk [14] showed that Theorem 1.9 can be significantly improved; namely, in the case $p<0$, each $f: E_{1} \rightarrow E_{2}$ satisfying (1.2) 
must actually be additive, and the assumption of completeness of $E_{2}$ is not necessary. Unfortunately, this result does not remain valid if we restrict the domain of $f$ (see the further detail in [16]). On the other hand, several mathematicians showed that the fixed point method is an another very efficient and convenient tool for proving the Hyers-Ulam stability for a quite wide class of functional equations (see [15]). J. Brzdȩk et al. [10] proved the fixed point theorem for a nonlinear operator in metric spaces and used this result to study the Hyers-Ulam stability of some functional equations in non-Archimedean metric spaces. In this work, they also obtained the fixed point result in arbitrary metric spaces as follows:

By using this theorem, Brzdęk [13] improved, extended and complemented several earlier classical stability results concerning the additive Cauchy equation (in particular Theorem 1.9). During the past few years many mathematicians have investigated various generalizations, extensions and applications of the Hyers-Ulam stability of a number of functional equations (see, for instance, $[1,2,3,5,6,15,16,12]$ and references therein).

Now, we present the fixed point theorem concerning 2-Banach spaces given in [11]. First, we need the following hypotheses:

(H1) $E$ is a nonempty set, $(Y,\|\cdot, \cdot\|)$ is a 2 -Banach space, $Y_{0}$ is a subset of $Y$ containing two linearly independent vectors, $j \in \mathbf{N}, f_{i}: E \rightarrow E$, $g_{i}: Y_{0} \rightarrow Y_{0}$, and $L_{i}: E \times Y_{0} \rightarrow \mathbf{R}_{+}$for $i=1, \ldots, j$;

(H2) $\mathcal{T}: Y^{E} \rightarrow Y^{E}$ is an operator satisfying the inequality

$\|\mathcal{T} \xi(x)-\mathcal{T} \mu(x), y\| \leq \sum_{i=1}^{j} L_{i}(x, y)\left\|\xi\left(f_{i}(x)\right)-\mu\left(f_{i}(x)\right), g_{i}(y)\right\|, \xi, \mu \in Y^{E}, x \in E, y \in Y_{0} ;$

(H3) $\Lambda: \mathbf{R}_{+}^{E \times Y_{0}} \rightarrow \mathbf{R}_{+}^{E \times Y_{0}}$ is an operator defined by

$(1.5) \Lambda \delta(x, y):=\sum_{i=1}^{j} L_{i}(x, y) \delta\left(f_{i}(x), g_{i}(y)\right), \quad \delta \in \mathbf{R}_{+}^{E \times Y_{0}}, x \in E, y \in Y_{0}$.

Theorem 1.10. [11] Let hypotheses (H1)-(H3) hold and functions $\varepsilon: E \times$ $Y_{0} \rightarrow R_{+}$and $\varphi: E \rightarrow Y$ fulfill the following two conditions:

$$
\|\mathcal{T} \varphi(x)-\varphi(x), y\| \leq \varepsilon(x, y) \quad x \in E, y \in Y_{0},
$$




$$
\varepsilon^{*}(x, y):=\sum_{n=0}^{\infty}\left(\Lambda^{n} \varepsilon\right)(x, y)<\infty \quad x \in E, y \in Y_{0} .
$$

Then, there exists a unique fixed point $\psi$ of $\mathcal{T}$ for which

$$
\|\varphi(x)-\psi(x), y\| \leq \varepsilon^{*}(x, y) \quad x \in E, y \in Y_{0}
$$

Moreover,

$$
\psi(x):=\lim _{n \rightarrow \infty}\left(\mathcal{T}^{n} \varphi\right)(x) \quad x \in E .
$$

\section{Main results}

In this section, we prove some stability and hyperstability results for the Cauchy-Jensen equation (1.1) in 2-Banach spaces by using Theorem 1.10. In what follows $(Y,\|\cdot, \cdot\|)$ is a real 2 -Banach space.

Theorem 2.1. Let $h_{1}, h_{2}, h_{3}: E_{0} \times Y_{0} \rightarrow \mathbf{R}_{+}$be three functions such that

$\mathcal{U}:=\left\{n \in \mathbf{N}: \alpha_{n}:=\lambda_{1}(1+n) \lambda_{2}(1+n) \lambda_{3}(1+n)+\lambda_{1}(n) \lambda_{2}(n) \lambda_{3}(n)<1\right\} \neq \phi$

where

$$
\lambda_{i}(n):=\inf \left\{t \in \mathbf{R}_{+}: h_{i}(n x, w) \leq t h_{i}(x, w), \quad x \in E_{0}, w \in Y_{0}\right\}
$$

for all $n \in \mathbf{N}$, where $i=1,2,3$. Assume that $f: E \rightarrow Y$ satisfies the inequality

$\left\|f\left(\frac{x+y}{2}+z\right)+f\left(\frac{x-y}{2}+z\right)-2 f(z)-f(x), w\right\| \leq h_{1}(x, w) h_{2}(y, w) h_{3}(z, w)$,

for all $x, y, z, \in E_{0}, w \in Y_{0}$ such that $\frac{x+y}{2}+z \neq 0$ and $\frac{x-y}{2}+z \neq 0$. Then there exists a unique Cauchy-Jensen function $F: E \rightarrow Y$ such that 


$$
\|f(x)-F(x), w\| \leq \lambda_{0} h_{1}(x, w) h_{2}(x, w) h_{3}(x, w)
$$

for all $x \in E_{0}, w \in Y_{0}$, where

$$
\lambda_{0}:=\inf _{n \in \mathcal{U}}\left\{\frac{\lambda_{1}(n) \lambda_{2}(n)}{1-\lambda_{1}(1+n) \lambda_{2}(1+n) \lambda_{3}(1+n)-\lambda_{1}(n) \lambda_{2}(n) \lambda_{3}(n)}\right\} .
$$

Proof. Replacing $y$ and $x$ with $m x$ and $z$ with $x$, where $x \in E_{0}$ and $m \in \mathbf{N}$, in inequality (2.3) we get

$$
\|f((1+m) x)-f(m x)-f(x), w\| \leq h_{1}(m x, w) h_{2}(m x, w) h_{3}(x, w)
$$

for all $x \in E_{0}, w \in Y_{0}$. For each $m \in \mathbf{N}$, we define the operator $\mathcal{T}_{m}: Y^{E_{0}} \rightarrow$ $Y^{E_{0}}$ by

$$
\mathcal{T}_{m} \xi(x):=\xi((1+m) x)-\xi(m x), \quad \xi \in Y^{E_{0}}, x \in E_{0} .
$$

Further put

$$
\varepsilon_{m}(x, w):=h_{1}(m x, w) h_{2}(m x, w) h_{3}(x, w), \quad x \in E_{0}, w \in Y_{0},
$$

and observe that

$\varepsilon_{m}(x, w)=h_{1}(m x, w) h_{2}(m x, w) h_{3}(x, w) \leq \lambda_{1}(m) \lambda_{2}(m) h_{1}(x, w) h_{2}(x, w) h_{3}(x, w)$, (2.8)

for all $x \in E_{0}, w \in Y_{0}, m \in \mathbf{N}$. Then the inequality (2.5) takes the form

$$
\left\|f(x)-\mathcal{T}_{m} f(x), w\right\| \leq \varepsilon_{m}(x, w), \quad x \in E_{0}, w \in Y_{0} .
$$

Furthermore, for every $x \in E_{0}, w \in Y_{0}, \xi, \mu \in Y^{E_{0}}$, we obtain

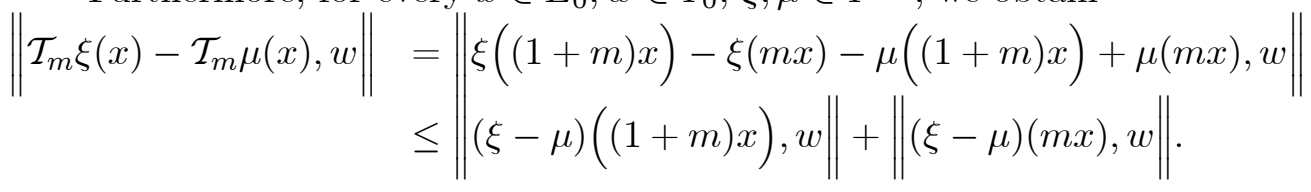

So, (H2) is valid for $\mathcal{T}_{m}$.

This brings us to define the operator $\Lambda_{m}: \mathbf{R}_{+}^{E_{0} \times Y_{0}} \rightarrow \mathbf{R}_{+}^{E_{0} \times Y_{0}}$ by

$$
\Lambda_{m} \delta(x, w):=\delta((1+m) x, w)+\delta(m x, w), \quad \delta \in \mathbf{R}_{+}^{E_{0} \times Y_{O}}, x \in E_{0}, w \in Y_{0}
$$


For each $m \in \mathbf{N}$, the above operator has the form described in (H3) with $f_{1}(x)=(1+m) x, f_{2}(x)=m x, g_{1}(w)=g_{2}(w)=w$ and $L_{1}(x)=L_{2}(x)=1$ for all $x \in E_{0}$. By induction, we will show that for each $x \in E_{0}, w \in Y_{0}$, $n \in \mathbf{N}_{0}$, and $m \in \mathcal{U}$ we have

$$
\left(\Lambda_{m}^{n} \varepsilon_{m}\right)(x, w) \leq \lambda_{1}(m) \lambda_{2}(m) \alpha_{m}^{n} h_{1}(x, w) h_{2}(x, w) h_{3}(x, w)
$$

where

$$
\alpha_{m}=\lambda_{1}(1+m) \lambda_{2}(1+m) \lambda_{3}(1+m)+\lambda_{1}(m) \lambda_{2}(m) \lambda_{3}(m) .
$$

From (2.7) and (2.8), we obtain that the inequality (2.11) holds for $n=0$. Next, we will assume that (2.11) holds for $n=k$, where $k \in \mathbf{N}$.

Then we have

$$
\begin{aligned}
\left(\Lambda_{m}^{k+1} \varepsilon_{m}\right)(x, w)= & \Lambda_{m}\left(\left(\Lambda_{m}^{k} \varepsilon_{m}\right)(x, w)\right) \\
= & \left(\Lambda_{m}^{k} \varepsilon_{m}\right)((1+m) x, w)+\left(\Lambda_{m}^{k} \varepsilon_{m}\right)(m x, w) \\
\leq & \lambda_{1}(m) \lambda_{2}(m) \alpha_{m}^{k} h_{1}((1+m) x, w) h_{2}((1+m) x, w) h_{3}((1+m) x, w) \\
& +\lambda_{1}(m) \lambda_{2}(m) \alpha_{m}^{k} h_{1}(m x, w) h_{2}(m x, w) h_{3}(m x, w) \\
\leq & \lambda_{1}(m) \lambda_{2}(m) \alpha_{m}^{k+1} h_{1}(x, w) h_{2}(x, w) h_{3}(x, w)
\end{aligned}
$$

for all $x \in E_{0}, w \in Y_{0}, m \in \mathcal{U}$. This shows that (2.11) holds for $n=k+1$. Now we can conclude that the inequality (2.11) holds for all $n \in \mathbf{N}_{0}$. Hence, we obtain

$$
\begin{aligned}
\varepsilon_{m}^{*}(x, w) & =\sum_{n=0}^{\infty}\left(\Lambda_{m}^{n} \varepsilon_{m}\right)(x, w) \\
& \leq \sum_{n=0}^{\infty} \lambda_{1}(m) \lambda_{2}(m) \alpha_{m}^{n} h_{1}(x, w) h_{2}(x, w) h_{3}(x, w) \\
& =\frac{\lambda_{1}(m) \lambda_{2}(m)}{1-\alpha_{m}} h_{1}(x, w) h_{2}(x, w) h_{3}(x, w)<\infty
\end{aligned}
$$

for all $x \in E_{0}, w \in Y_{0}, m \in \mathcal{U}$. Therefore, according to Theorem 1.10 with $\varphi=f$, we get that the limit

$$
F_{m}(x):=\lim _{n \rightarrow \infty}\left(\mathcal{T}_{m}^{n} f\right)(x)
$$

exists for each $x \in E_{0}$ and $m \in \mathcal{U}$, and

$$
\left\|f(x)-F_{m}(x), w\right\| \leq \frac{\lambda_{1}(m) \lambda_{2}(m) h_{1}(x, w) h_{2}(x, w) h_{3}(x, w)}{1-\alpha_{m}}, \quad x \in E_{0}, w \in Y_{0}, m \in \mathcal{U}
$$


To prove that $F_{m}$ satisfies the functional equation (1.1), just prove the following inequality

$$
\begin{aligned}
& \left\|\left(\mathcal{T}_{m}^{n} f\right)\left(\frac{x+y}{2}+z\right)+\left(\mathcal{T}_{m}^{n} f\right)\left(\frac{x-y}{2}+z\right)-2\left(\mathcal{T}_{m}^{n} f\right)(z)-\left(\mathcal{T}_{m}^{n} f\right)(x), w\right\| \\
& \leq \alpha_{m}^{n} h_{1}(x, w) h_{2}(y, w) h_{3}(z, w)
\end{aligned}
$$

for every $x, y, z \in E_{0}$ such that $\frac{x+y}{2}+z \neq 0$ and $\frac{x-y}{2}+z \neq 0, w \in Y_{0}, n \in \mathbf{N}_{0}$, and $m \in \mathcal{U}$. Since the case $n=0$ is just (2.3), take $k \in \mathbf{N}$ and assume that (2.13) holds for $n=k$ and every $x, y, z \in E_{0}$ such that $\frac{x+y}{2}+z \neq 0$ and $\frac{x-y}{2}+z \neq 0, w \in Y_{0}, m \in \mathcal{U}$. Then, for each $x, y, z \in E_{0}, w \in Y_{0}$ and $m \in \mathcal{U}$, we get

$$
\begin{aligned}
& \left\|\left(\mathcal{T}_{m}^{k+1} f\right)\left(\frac{x+y}{2}+z\right)+\left(\mathcal{T}_{m}^{k+1} f\right)\left(\frac{x-y}{2}+z\right)-2\left(\mathcal{T}_{m}^{k+1} f\right)(z)-\left(\mathcal{T}_{m}^{k+1} f\right)(x), w\right\| \\
& =\|\left(\mathcal{T}_{m}^{k} f\right) f\left((1+m)\left(\frac{x+y}{2}+z\right)\right)-\left(\mathcal{T}_{m}^{k} f\right) f\left(m\left(\frac{x+y}{2}+z\right)\right) \\
& +\left(\mathcal{T}_{m}^{k} f\right) f\left((1+m)\left(\frac{x-y}{2}+z\right)\right)-\left(\mathcal{T}_{m}^{k} f\right) f\left(m\left(\frac{x-y}{2}+z\right)\right) \\
& -2\left(\mathcal{T}_{m}^{k} f\right) f((1+m) z)+2\left(\mathcal{T}_{m}^{k} f\right) f(m z)-\left(\mathcal{T}_{m}^{k} f\right) f((1+m) x)+\left(\mathcal{T}_{m}^{k} f\right) f(m x), w \| \\
& \leq \|\left(\mathcal{T}_{m}^{k} f\right) f\left((1+m)\left(\frac{x+y}{2}+z\right)\right)+\left(\mathcal{T}_{m}^{k} f\right) f\left((1+m)\left(\frac{x-y}{2}+z\right)\right) \\
& -2\left(\mathcal{T}_{m}^{k} f\right) f((1+m) z)-\left(\mathcal{T}_{m}^{k} f\right) f((1+m) x), w \|+ \\
& \left\|\left(\mathcal{T}_{m}^{k} f\right) f\left(m\left(\frac{x+y}{2}+z\right)\right)+\left(\mathcal{T}_{m}^{k} f\right) f\left(m\left(\frac{x-y}{2}+z\right)\right)-2\left(\mathcal{T}_{m}^{k} f\right) f(m z)-\left(\mathcal{T}_{m}^{k} f\right) f(m x), w\right\| \\
& \leq \alpha_{m}^{k} h_{1}((1+m) x, w) h_{2}((1+m) y, w) h_{3}((1+m) z, w)+ \\
& \alpha_{m}^{k} h_{1}(m x, w) h_{2}(m y, w) h_{3}(m z, w) \\
& \leq \alpha_{m}^{k+1} h_{1}(x, w) h_{2}(y, w) h_{3}(z, w)
\end{aligned}
$$

Thus, by induction, we have shown that (2.13) holds for every $x, y, z \in$ $E_{0}$ such that $\frac{x+y}{2}+z \neq 0$ and $\frac{x-y}{2}+z \neq 0, w \in Y_{0}, n \in \mathbf{N}_{0}$, and $m \in \mathcal{U}$. Letting $n \rightarrow \infty$ in (2.13), we obtain the equality

$$
F_{m}\left(\frac{x+y}{2}+z\right)+F_{m}\left(\frac{x-y}{2}+z\right)=F_{m}(x)+2 F_{m}(z)
$$


for all $x, y, z \in E_{0}$ such that $\frac{x+y}{2}+z \neq 0$ and $\frac{x-y}{2}+z \neq 0, m \in \mathcal{U}_{\text {l. This }}$ implies that $F_{m}: E_{0} \rightarrow Y$, defined in this way, is a solution of the equation

$$
F(x)=F((1+m) x)-F(m x), \quad x \in E_{0}, m \in \mathcal{U}
$$

Next, we will prove that each Cauchy-Jensen function $F: E \rightarrow Y$ satisfying the inequality

$$
\|f(x)-F(x), w\| \leq L h_{1}(x, w) h_{2}(x, w) h_{3}(x, w), \quad x \in E_{0}, w \in Y_{0}
$$

with some $L>0$, is equal to $F_{m}$ for each $m \in \mathcal{U}$. To this end, we fix $m_{0} \in \mathcal{U}$ and $F: E \rightarrow Y$ satisfying (2.16). From (2.12), for each $x \in E_{0}$, we get

$$
\begin{aligned}
\left\|F(x)-F_{m_{0}}(x), w\right\| & \leq\|F(x)-f(x), w\|+\left\|f(x)-F_{m_{0}}(x), w\right\| \\
& \leq L h_{1}(x, w) h_{2}(x, w) h_{3}(x, w)+\varepsilon_{m_{0}}^{*}(x, w) \\
& \leq L_{0} h_{1}(x, w) h_{2}(x, w) h_{3}(x, w) \sum_{n=0}^{\infty} \alpha_{m_{0}}^{n}
\end{aligned}
$$

where

$L_{0}:=\left(1-\alpha_{m_{0}}\right) L+\lambda_{1}\left(m_{0}\right) \lambda_{2}\left(m_{0}\right)>0$ and we exclude the case that $h_{1}(x, w) \equiv 0, h_{2}(x, w) \equiv 0$ or $h_{3}(x, w) \equiv 0$ which is trivial. Observe that $F$ and $F_{m_{0}}$ are solutions to equation (2.15) for all $m \in \mathcal{U}$. Next, we show that, for each $j \in \mathbf{N}_{0}$, we have

$$
\left\|F(x)-F_{m_{0}}(x), w\right\| \leq L_{0} h_{1}(x, w) h_{2}(x, w) h_{3}(x, w) \sum_{n=j}^{\infty} \alpha_{m_{0}}^{n}, \quad x \in E_{0}, w \in Y_{0} .
$$

The case $j=0$ is exactly (2.17). We fix $k \in \mathbf{N}$ and assume that (2.18) holds for $j=k$. Then, in view of (2.17), for each $x \in E_{0}, w \in Y_{0}$, we get 


$$
\begin{aligned}
\left\|F(x)-F_{m_{0}}(x), w\right\|= & \| F\left(\left(1+m_{0}\right) x\right)-F\left(m_{0} x\right) \\
- & F_{m_{0}}\left(\left(1+m_{0}\right) x\right)+F_{m_{0}}\left(m_{0} x\right), w \| \\
\leq & \left\|F\left(\left(1+m_{0}\right) x\right)-F_{m_{0}}\left(\left(1+m_{0}\right) x\right), w\right\| \\
& +\left\|F\left(m_{0} x\right)-F_{m_{0}}\left(m_{0} x\right), w\right\| \\
\leq & L_{0} h_{1}\left(\left(1+m_{0}\right) x, w\right) h_{2}\left(\left(1+m_{0}\right) x, w\right) h_{3}\left(\left(1+m_{0}\right) x, w\right) \sum_{n=k}^{\infty} \alpha_{m_{0}}^{n} \\
& +L_{0} h_{1}\left(m_{0} x, w\right) h_{2}\left(m_{0} x, w\right) h_{3}\left(m_{0} x, w\right) \sum_{n=k}^{\infty} \alpha_{m_{0}}^{n} \\
= & L_{0}\left(h_{1}\left(\left(1+m_{0}\right) x, w\right) h_{2}\left(\left(1+m_{0}\right) x, w\right) h_{3}\left(\left(1+m_{0}\right) x, w\right)\right. \\
& \left.+h_{1}\left(m_{0} x, w\right) h_{2}\left(m_{0} x, w\right) h_{3}\left(m_{0} x, w\right)\right) \sum_{n=k}^{\infty} \alpha_{m_{0}}^{n} \\
\leq & L_{0} \alpha_{m_{0}} h_{1}(x, w) h_{2}(x, w) h_{3}(x, w) \sum_{n=k}^{\infty} \alpha_{m_{0}}^{n} \\
= & L_{0} h_{1}(x, w) h_{2}(x, w) h_{3}(x, w) \sum_{n=k+1}^{\infty} \alpha_{m_{0}}^{n} .
\end{aligned}
$$

This shows that (2.18) holds for $j=k+1$. Now we can conclude that the inequality (2.18) holds for all $j \in \mathbf{N}_{0}$. Now, letting $j \rightarrow \infty$ in (2.18), we get

$$
F=F_{m_{0}} .
$$

Thus, we have also proved that $F_{m}=F_{m_{0}}$ for each $m \in \mathcal{U}$, which (in view of (2.12)) yields

$$
\left\|f(x)-F_{m_{0}}(x), w\right\| \leq \frac{\lambda_{1}(m) \lambda_{2}(m) h_{1}(x, w) h_{2}(x, w) h_{3}(x, w)}{1-\alpha_{m}}, x \in E_{0}, w \in Y_{0}, m \in \mathcal{U}
$$

This implies (2.4) with $F=F_{m_{0}}$ and (2.19) confirms the uniqueness of $F$.

The following theorem concerns the $\eta$-hyperstability of (1.1) in 2-Banach spaces. Namely, We consider functions $f: E \rightarrow Y$ fulfilling (1.1) approximately, i.e., satisfying the inequality

$$
\left\|f\left(\frac{x+y}{2}+z\right)+f\left(\frac{x-y}{2}+z\right)-2 f(z)-f(x), w\right\| \leq \eta(x, y, z, w),
$$

for all $x, y, z \in E_{0}$ such that $\frac{x+y}{2}+z \neq 0$ and $\frac{x-y}{2}+z \neq 0, w \in Y_{0}$, with $\eta: E_{0} \times E_{0} \times E_{0} \times Y_{0} \rightarrow \mathbf{R}_{+}$is a given mapping. Then we find a unique Cauchy-Jensen function $F: E \rightarrow Y$ which is close to $f$. Then, under 
some additional assumptions on $\eta$, we prove that the conditional functional equation (1.1) is $\eta$-hyperstable in the class of functions $f: E \rightarrow Y$, i.e., each $f: E \rightarrow Y$ satisfying inequality (2.21), with such $\eta$, must fulfil equation (1.1).

Theorem 2.2. Let $h_{1}, h_{2}, h_{3}$ and $\mathcal{U}$ be as in Theorem 2.1. Assume that

$$
\left\{\begin{array}{l}
\lim _{n \rightarrow \infty} \lambda_{1}(n) \lambda_{2}(n)=0, \\
\lim _{n \rightarrow \infty} \lambda_{1}(n) \lambda_{2}(n) \lambda_{3}(n)=0 .
\end{array}\right.
$$

Then every $f: E \rightarrow Y$ satisfying (2.3) is a solution of (1.1).

Proof. Suppose that $f: E \rightarrow Y$ satisfies (2.3). Then, by Theorem 2.1, there exists a mapping $F: E \rightarrow Y$ satisfies (1.1)

and

$$
\|f(x)-F(x), w\| \leq \lambda_{0} h_{1}(x, w) h_{2}(x, w) h_{3}(x, w)
$$

for all $x \in E_{0}, w \in Y_{0}$, where

$$
\lambda_{0}:=\inf _{n \in \mathcal{U}}\left\{\frac{\lambda_{1}(n) \lambda_{2}(n)}{1-\lambda_{1}(1+n) \lambda_{2}(1+n) \lambda_{3}(1+n)-\lambda_{1}(n) \lambda_{2}(n) \lambda_{3}(n)}\right\} .
$$

Since, in view of $(2.22), \lambda_{0}=0$. This means that $f(x)=F(x)$ for all $x \in E_{0}$, whence

$$
f\left(\frac{x+y}{2}+z\right)+f\left(\frac{x-y}{2}+z\right)=f(x)+2 f(z)
$$

for all $x, y, z \in E_{0}$ such that $\frac{x+y}{2}+z \neq 0$ and $\frac{x-y}{2}+z \neq 0$, which implies that $f$ satisfies the functional equation (1.1) on $E$.

Corollary 2.3. Let $\theta \geq 0, s \geq 0, p, q, r \in \mathbf{R}$ such that $p+q+r<0$. Suppose that $f: E \rightarrow Y$ such that $f(0)=0$ satisfy the inequality

$$
\left\|f\left(\frac{x+y}{2}+z\right)+f\left(\frac{x-y}{2}+z\right)-2 f(z)-f(x), w\right\| \leq \theta\|x\|^{p}\|y\|^{q}\|z\|^{r}\|w\|^{s},
$$

for all $x, y, z \in E_{0}$ such that $\frac{x+y}{2}+z \neq 0$ and $\frac{x-y}{2}+z \neq 0, w \in Y_{0}$. Then $f$ is Cauchy-Jensen on $E_{0}$. 
Proof. The proof follows from Theorem 2.1 by defining

$h_{1}, h_{2}, h_{3}: E_{0} \times Y_{0} \rightarrow \mathbf{R}_{+}$by $h_{1}(x, w)=\theta_{1}\|x\|^{p}\|w\|^{s_{1}}, h_{2}(y, w)=\theta_{2}|y|^{q}|w|^{s_{2}}$, $h_{3}(z, w)=\theta_{3}\|z\|^{r}\|w\|^{s_{3}}$ and $h_{1}(0, w)=h_{2}(0, w)=h_{3}(0, w)=0$ with $\theta_{1}, \theta_{2}, \theta_{3} \in \mathbf{R}_{+}, s_{1}, s_{2}, s_{3} \in \mathbf{R}_{+}$and $p, q, r \in \mathbf{R}$ such that $\theta_{1} \theta_{2} \theta_{3}=\theta$, $s_{1}+s_{2}+s_{3}=s$ and $p+q+r<0$.

For each $n \in \mathbf{N}$, we have

$$
\begin{aligned}
\lambda_{1}(n) & =\inf \left\{t \in \mathbf{R}_{+}: h_{1}(n x, w) \leq t h_{1}(x, w), \quad x \in E_{0}, w \in Y_{0}\right\} \\
& =\inf \left\{t \in \mathbf{R}_{+}: \theta_{1}\|n x\|^{p}\|w\|^{s_{1}} \leq t \theta_{1}\|x\|^{p}\|w\|^{s_{1}}, \quad x \in E_{0}, w \in Y_{0}\right\} \\
& =n^{p}
\end{aligned}
$$

Also, we have $\lambda_{2}(n)=n^{q}$ and $\lambda_{3}(n)=n^{r}$ for all $n \in \mathbf{N}$. Clearly, we can find $n_{0} \in \mathbf{N}$ such that

$\lambda_{1}(1+n) \lambda_{2}(1+n) \lambda_{3}(1+n)+\lambda_{1}(n) \lambda_{2}(n) \lambda_{3}(n)=(1+n)^{p+q+r}+n^{p+q+r}<1, \quad n \geq n_{0}$.

According to Theorem 2.1, there exists a unique Cauchy-Jensen function $F: E \rightarrow Y$ such that

$$
\|f(x)-F(x), w\| \leq \theta \lambda_{0} h_{1}(x, w) h_{2}(x, w) h_{3}(x, w)
$$

for all $x \in E_{0}, w \in Y_{0}$, where

$$
\lambda_{0}:=\inf _{n \in \mathcal{U}}\left\{\frac{\lambda_{1}(n) \lambda_{2}(n)}{1-\lambda_{1}(1+n) \lambda_{2}(1+n) \lambda_{3}(1+n)-\lambda_{1}(n) \lambda_{2}(n) \lambda_{3}(n)}\right\} .
$$

On the other hand, Since $p+q+r<0$, It is sufficient to consider that $p+q<0$. Then

$$
\left\{\begin{array}{l}
\lim _{n \rightarrow \infty} \lambda_{1}(n) \lambda_{2}(n)=\lim _{n \rightarrow \infty} n^{p+q}=0, \\
\lim _{n \rightarrow \infty} \lambda_{1}(n) \lambda_{2}(n) \lambda_{3}(n)=\lim _{n \rightarrow \infty} n^{p+q+r}=0 .
\end{array}\right.
$$

Thus by Theorem 2.2, we get the desired results.

The next corollary prove the hyperstability results for the inhomogeneous Cauchy-Jensen functional equation.

Corollary 2.4. Let $\theta, p, q, r, s \in \mathbf{R}$ such that $\theta \geq 0$ and $p+q+r<0$. Assume that $G: E^{3} \rightarrow Y$ and $f: E \rightarrow Y$ such that $f(0)=0$ and satisfy the inequality 


$$
\left\|f\left(\frac{x+y}{2}+z\right)+f\left(\frac{x-y}{2}+z\right)-2 f(z)-f(x)-G(x, y, z), w\right\| \leq \theta\|x\|^{p}\|y\|^{q}\|z\|^{r}\|w\|^{s}
$$

for all $x, y, z \in E_{0}$ such that $\frac{x+y}{2}+z \neq 0$ and $\frac{x-y}{2}+z \neq 0, w \in Y_{0}$. If the functional equation

$$
f\left(\frac{x+y}{2}+z\right)+f\left(\frac{x-y}{2}+z\right)=2 f(z)+f(x)+G(x, y, z)
$$

for all $x, y, z \in E_{0}$ such that $\frac{x+y}{2}+z \neq 0$ and $\frac{x-y}{2}+z \neq 0$, has a solution $f_{0}: E \rightarrow Y$, then $f$ is a solution to (2.29).

Proof. From (2.28) we get that the function $K: E \rightarrow Y$ defined by $K:=f-f_{0}$ satisfies (2.24). Consequently, Corollary 2.3 implies that $K$ is a solution to Cauchy-Jensen functional equation (1.1). Therefore,

$$
\begin{aligned}
& f\left(\frac{x+y}{2}+z\right)+f\left(\frac{x-y}{2}+z\right)-2 f(z)-f(x)-G(x, y, z)=K\left(\frac{x+y}{2}+z\right) \\
& \quad+f_{0}\left(\frac{x+y}{2}+z\right)+K\left(\frac{x-y}{2}+z\right)+f_{0}\left(\frac{x-y}{2}+z\right) \\
& \quad-2 K(z)-2 f_{0}(z)-K(x)-f_{0}(x)-G(x, y, z) \\
& \quad=0
\end{aligned}
$$

for all $x, y, z \in E_{0}$ such that $\frac{x+y}{2}+z \neq 0$ and $\frac{x-y}{2}+z \neq 0$, which means $f$ is a solution to (2.29).

\section{References}

[1] M. Almahalebi and C. Park, "On the hyperstability of a functional equation in commutative groups", Journal of computational analysis and applications, vol. 20, no. 1, pp. 826-833, 2016.

[2] M. Almahalebi and A. Chahbi, "Hyperstability of the Jensen functional equation in ultrametric spaces", Aequationes mathematicae, vol. 91, no. 4, pp. 647-661, Aug. 2017, doi: 10.1007/s00010-017-0487-6.

[3] M. Almahalebi, "Non-Archimedean hyperstability of a Cauchy-Jensen type functional equation", Journal of classical analysis, vol. 11, no. 2, pp. 159- 170, Oct. 2017, doi: 10.7153/jca-2017-11-10. 
[4] T. Aoki, "On the stability of the linear transformation in Banach spaces", Journal of the mathematical society of Japan, vol. 2, no. 1-2, pp. 64-66, 1950, doi: 10.2969/jmsj/00210064.

[5] L. Aiemsomboon and W. Sintunavarat, "On new stability results for generalized Cauchy functional equations on groups by using Brzdęk's fixed point theorem", Journal of fixed point theory and applications, vol. 18, no. 1, pp. 45-59, Mar. 2016, doi: 10.1007/s11784-015-0259-7.

[6] L. Aiemsomboon and W. Sintunavarat, "On generalized hyperstability of a general linear equation", Acta mathematica hungarica, vol. 149, no. 2, pp. 413-422, Aug. 2016, doi: 10.1007/s10474-016-0621-2.

[7] C. Baak, "Cauchy-Rassias stability of Cauchy-Jensen additive mappings in Banach spaces", Acta mathematica sinica, vol. 22, no. 6, pp. 17891796, Nov. 2006, doi: 10.1007/s10114-005-0697-z.

[8] D. G. Bourgin, "Classes of transformations and bordering transformations", Bulletin of the American mathematical society, vol. 57, no. 4, pp. 223-237, 1951, doi: 10.1090/S0002-9904-1951-09511-7.

[9] J. Brzdęk, "A note on stability of additive mappings," in Stability of mappings of Hyers-Ulam type, T. M. Rassias and J. Tabor, Eds. Palm Harbor, FL: Hadronic Press, 1994, pp. 19-22.

[10] J. Brzdęk and K. Ciepliński, "A fixed point approach to the stability of functional equations in non-Archimedean metric spaces", Nonlinear analysis: theory, methods \& applications, vol. 74, no. 18, pp. 6861-6867, Dec. 2011, doi: 10.1016/j.na.2011.06.050.

[11] J. Brzdęk and K. Ciepliński, "On a fixed point theorm in 2-Banach spaces and some of its applications", Acta mathematica scientia, vol. 38, no. 2, pp. 377-390, Mar. 2018, doi: 10.1016/S0252-9602(18)30755-0.

[12] J. Brzdęk, D. Popa, I. Raşa, B. Xu, Ulam stability of operators, Amsterdam: Academic press, 2018, doi: 10.1016/C2015-0-06292-X.

[13] J. Brzdęk, "Stability of additivity and fixed point methods", Fixed point theory and applications, Art. ID. 265, 2013, doi: 10.1186/1687-1812-2013-285.

[14] J. Brzdęk, "Hyperstability of the Cauchy equation on restricted domains", Acta mathematica hungarica, vol. 141, no. 1-2, pp. 58-67, Oct. 2013, doi:10.1007/s10474-013-0302-3.

[15] J. Brzdęk, L. Cadăriu and K. Ciepliński, "Fixed point theory and the Ulam stability", Journal of function spaces, Art. ID 829419, Aug. 2014, doi:10.1155/2014/829419. 
[16] J. Brzdęk, W. Fechner, M. S. Moslehian and J. Sikorska, "Recent developments of the conditional stability of the homomorphism equation", Banach journal of mathematical analysis, vol. 9, no. 3, pp. 278-327, 2015, doi: 10.15352/bjma/09-3-20.

[17] R. W. Freese and Y. J. Cho, Geometry of linear 2-normed spaces. Hauppauge, NY: Nova Science Publishers, Inc., 2001.

[18] D. H. Hyers, "On the stability of the linear functional equation", Proceedings of the national academy of sciences of the United States of America, vol. 27, no. 4, pp. 222-224, Apr. 1941. [On line]. Available: https://bit.ly/2TUR5DN

[19] W. G. Park, "Approximate additive mappings in 2-Banach spaces and related topics", Journal of mathematical analysis and applications, vol. 376 , no. 1, pp. 193-202, Apr. 2011, doi: 10.1016/j.jmaa.2010.10.004.

[20] C. Park, "Fixed points and Hyers-Ulam-Rassias stability of Cauchy Jensen functional equations in Banach algebras", Fixed point theory and applications, Art. ID. 050175, Dec. 2007, doi: 10.1155/2007/50175.

[21] Th. M. Rassias, "On the stability of the linear mapping in Banach spaces", Proceedings of the American mathematical society, vol. 72, pp. 297-300, 1978, doi: 10.1090/S0002-9939-1978-0507327-1.

[22] Th. M. Rassias, "Problem 16, $2^{\circ}$. The Twenty-seventh International Symposium on Functional Equations, August 14-24, 1989, Bielsko-BiałKatowice-Kraków, Poland", Aequationes mathematicae, vol. 39, no. 2-3, pp. 292-293, Apr. 1990, doi: 10.1007/BF01833155.

[23] Th. M. Rassias, "On a modified Hyers-Ulam sequence”, Journal of mathematical analysis and applications, vol. 158, no. 1, pp. 106-113, Jun. 1991, doi: 10.1016/0022-247X(91)90270-A.

[24] S. M. Ulam, Problems in modern mathematics. New York, NY: John Wiley \& Sons, 1964. 